\title{
SILICOSIS COMO POSIBLE CONFUSOR DE METÁSTASIS PULMONARES EN PET/CT EN PACIENTE CON CÁNCER DE RECTO
}

Quijano Diana Carolina ${ }_{1}$, Izquierdo María Alejandra ${ }_{2}$, Varela Humberto ${ }_{3}$, Lutz Juan Ricardo 1. Médica residente de medicina nuclear, Fundación Universitaria Sanitas. 2. Médica hospitalaria, servicio de neumología HUM. 3. Médico Nuclear, INC. 4 . Médico Neumólogo, HUM.

\section{INTRODUCCIÓN}

La silicosis es una neumoconiosis que generalmente resulta de la exposición prolongada a sílice cristalino (SiO2), un compuesto inorgánico que se encuentra en productos naturales e industriales, tales como piedra, arena, concreto, ladrillos, entre otros. En Colombia los casos de neumoconiosis representan entre el $4 \%$ y el $9 \%$ del total de eventos de enfermedad profesional y la exposición a sílice puede llegar a afectar aproximadamente a 1.800 .000 trabajadores (1).

A continuación se describe el caso de un paciente del Hospital Universitario Mayor con cáncer de recto concomitante con silicosis y sospecha de metástasis

\section{OBJETIVOS}

Exponer los hallazgos gammagráficos de la silicosis conglomerada como un posible diagnóstico diferencial de metástasis pulmonares.

\section{MATERIALES Y MÉTODOS}

Se realiza descripción de un caso clínico y revisión no sistemática de la literatura médica utilizando los siguientes términos: Silicosis, neumoconiosis, cáncer de recto, metástasis

\section{RESULTADOS}

Se presenta el caso de un paciente masculino de 68 años con diagnóstico de adenocarcinoma de recto estado IV por hallazgos radiológicos de metástasis hepáticas. Entre los estudios de extensión iniciales, se realizó TC de tórax donde se documentaron ganglios mediastinales con calcificaciones en "cáscara de huevo" y nódulos que confluyen en los segmentos posteriores de los lóbulos superiores formando masas simétricas con densidad de tejidos blandos y nodularidad subpleural, interpretados como silicosis conglomerada (Figura 1). El paciente recibió esquema de quimioterapia con FOLFOX6-BEVACIZUMAB por 6 dosis, presentando respuesta clínica y paraclínica completa. Se realizó control post tratamiento con PET/CT con 18F-FDG, en el que persistía hallazgos descritos en TC de tórax inicial, con ganglios linfáticos mediastinales hipermetabólicos, lesión nodular hipermetabólica de bordes irregulares en el segmento posterior del lóbulo superior derecho con valor estandarizado de captación máximo (SUVmax) de 4.4 y en el segmento posterior del lóbulo superior izquierdo de $33 \times 26 \mathrm{~mm}$ con SUVmax de 10.8 (Figura 2). No se encontraron lesiones hepáticas y en recto presentó desaparición tumoral sin signos de recidiva. Se consideró que los hallazgos hipermetabólicos en lóbulo superior izquierdo podrian corresponder a metástasis pulmonar. Se llevo a junta de decisiones médicas interdisciplinarias que incluyó a radiología, coloproctología, neumología y oncología donde se discutieron los hallazgos tomográficos en tórax de tomografía previa y PET/CT, así como la relación entre los antecedentes exposicionales del paciente quien laboró en construcción con exposición a sílice sin elementos de protección personal.

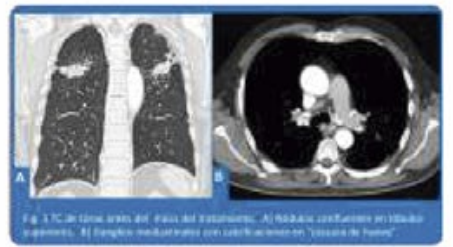

\section{CONCLUSIÓN}

El diagnóstico de silicosis es clínico, basándose en 3 criterios: historia de exposición a sílice cristalina, estudios radiológicos con hallazgos caracteristicos y exclusión de otro diagnóstico más probable. Hay que tener presente las patologías benignas que pueden dar nódulos hipermetabólicos al PET/CT para no confundirlas con metástasis; adicionalmente se debe realizar siempre seguimiento estricto y valorar al paciente de forma integral uniendo su clínica, antecedentes y estudios imagenológicos, para dar así un diagnóstico certero.
Se concluyó que los hallazgos imagenológicos y gammagráficos pulmonares eran concordantes con el diagnóstico de silicosis conglomerada. Se realizó TC de tórax de control al año y medio del diagnóstico inicial sin encontrar variación.

DISCUSIÓN

Las formas clínicas de la silicosis, clasificadas de acuerdo a su evolución temporal, son aguda, acelerada y crónica; y de acuerdo al compromiso parenquimatoso en simple, conglomerada o pseudotumoral y fibrosis masiva progresiva. La forma simple se caracteriza por micronódulos con predilección en lóbulos superiores, siendo la forma más frecuente. De haber una exposición a mayor cantidad de silice por un periodo de tiempo extenso, se puede observar la confluencia de dichos nódulos formando lesiones que se localizan en igual ubicación. Adicionalmente, en cualquiera de los estadios de la enfermedad se pueden encontrar adenopatías hiliares y mediastinales, que suelen calcificarse en la periferia, dando el patrón conocido como "calcificación en cáscara de huevo" (2). En el caso descrito tenemos un paciente con hallazgos imagenológicos típicos de silicosis en el contexto de una exposición conocida a sílice, quien presenta concomitante cáncer de recto estado IV tratado con quimioterapia, obteniendo respuesta completa, con desaparición de las lesiones hepáticas y del primario en recto, sin alteración alguna de lesiones pulmonares, con PET/CT de control post tratamiento que mostró lesiones pulmonares con gran avidez por la FDG de predominio en lóbulo superior izquierdo lo que hizo sospechar metástasis por la presencia de SUVmax $>8.0$ que da una probabilidad de malignidad de $96.2 \%$ (3). En la silicosis, se produce una reacción pulmonar parenquimatosa de tipo inflamatorio, compuesta por agregados de macrófagos, los cuales presentan captación de glucosa, siendo responsable del aumento del metabolismo y avidez por la FDG, mostrando nódulos hipermetabólicos en el PET/CT(4). Debemos sospechar en carcinoma pulmonar en paciente con silicosis cuando se presenta una masa unilateral y/o crecimiento rápido de las lesiones(2). En nuestro caso los hallazgos pulmonares eran bilaterales, simétricos y al seguimiento durante año y medio no presentó variación alguna.

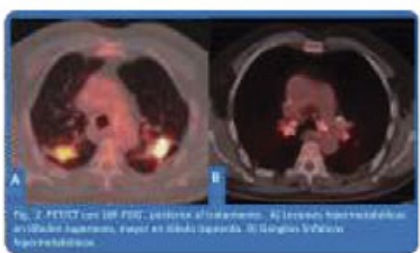

REFERENCIAS

1. Ministerio de la Protección Social, República de Colombia. (2010). Plan nacional para la prevención de la silicosis, la neumoconiosis de los mineros de carbón y la asbestosis 2010 - 2030.

2. González M, Trinidad C, Castellón D, et al. (2013). Silicosis pulmonar hallazgos radiológicos en la tomografía computarizada. Radiología, 55(6), 523-532, doi: $10.1016 / \mathrm{j} . \mathrm{rx} .2011 .12 .010$.

3. Hou $S$, Lin X, Wang $S$, et al. Combination of positron emission tomography/computed tomography and chest thin-layer high-resolution computed tomography for evaluation of pulmonary nodules: Correlation with imaging features, maximum standardized uptake value, and pathology. (2018). Medicine, 97(31), e11640. doi: $10.1097 / \mathrm{MD} .0000000000011640$

4. Capitanio S, Nordin AJ, Noraini AR, Rossetti C. (2016). PET/CT in nononcological lung diseases: current applications and future perspectives. European Respiratory Review, 25 (141), 247-258. doi: $10.1183 / 16000617.0051-2016$ 